Z Gerontol Geriat 2017 · 50:573-575 https://doi.org/10.1007/s00391-017-1317-0 Online publiziert: 21. September 2017 (c) Springer Medizin Verlag GmbH 2017

CrossMark

\author{
Walter Hewer ${ }^{1}$ Rüdiger Thiesemann ${ }^{2}$ \\ 'Klinikum Christophsbad, Göppingen, Deutschland \\ ${ }^{2}$ Wissenschaftliches Büro, Cuxhaven, Deutschland
}

\title{
Demenz als interdisziplinäres Thema von Alter(n)swissenschaft und Alter(n)smedizin
}

nung der zuerst genannten 270 Publikationen, die von den Verfassern in orientierender Form vorgenommen wurde, listet - Tab. 1 auf. Erwähnenswert ist daneben auch, dass sich in den zurückliegenden Jahren eine Reihe von Themenschwerpunkten mit unterschiedlichen Facetten des Generalthemas „Demenz“ beschäftigt hat (•Tab. 2).

Unsere Zeitschrift hat somit in den zurückliegenden Jahren deutlich gemacht, für wie wichtig sie die Herausforderungen hält, die aus der wachsenden Zahl der an Demenz Erkrankten in unserer Gesellschaft resultieren. Für das vorliegende Heft wurden aus der Fülle in Betracht kommender Themen einige Aspekte ausgewählt, von denen wir hoffen, dass sie für den Kreis unserer Leserinnen und Leser von besonderem Interesse sind und auch dem Format eines Jubiläumsheftes gerecht werden.

J. Pantel gibt einen historischen Überblick zur Entwicklung der pathogenetischen Konzepte der Alzheimer-Demenz. Er arbeitet heraus, dass diese Erkrankung über lange Zeit ein Nischendasein fristete, bis in den 80er Jahren des letzten Jahrhunderts erkannt wurde, dass das bis dahin üblicherweise als senile Demenz bezeichnete Störungsbild als eine spät auftretende Manifestation der Alzheimer-Demenz anzusehen ist und die ebenfalls in dieser Zeit sich abzeichnende enorme Zunahme der Prävalenz dieser Erkrankung offensichtlichen Handlungsbedarf erzeugte. Die in der Folge rasch anwachsenden weltweiten Forschungsaktivitäten erbrachten eine Vielzahl fruchtbarer Ergebnisse, und die Amyloid-Kaskaden-Hypothese etablierte sich als füh- rendes Paradigma der Alzheimer-Forschung. Gleichwohl bleibt eine Reihe von Fragen offen, sodass alternative pathogenetische Konzepte im Blick behalten werden müssen, ebenso wie derzeit noch keine zuverlässige Aussage dazu möglich ist, ob und wann krankheitsmodifizierende Therapien zur Verfügung stehen werden.

Gegenstand des Beitrags von L. Neumann, U. Dapp, W. Jacobsen und W. von Renteln-Kruse sind Ergebnisse aus dem MINDMAP-Projekt, einem internationalen longitudinalen Kohortenforschungsprojekt, welches aus der klinischen Geriatrie wesentliche Gründungsimpulse erhielt und in 12 Städten Europas gemeinsam durchgeführt wird. Ziel verschiedener MINDMAP-Projekte ist es - so die Autoren - u. a., ,eine geriatrische Perspektive zu entwickeln, um die Verbindung zwischen Umgebungen und mentalem Wohlbefinden einerseits und dem Frailty-Komplex" andererseits $\mathrm{zu}$ erhellen. Dieses dient dem Zweck, zielgruppengenaue Interventionen $\mathrm{zu}$ entwickeln, bevor eine Gebrechlichkeit einzutreten droht („pre-frailty“).

Hierzu wurde ein aufwendiger Survey entwickelt, der Datenerhebungen aus einer Vielzahl von Studienzentralen abrief. Hierbei wurden sowohl Public-HealthStrategien als auch klinisch geriatrische und epidemiologische Ansätze untersucht. Zentraler Aspekt dieser Strategien ist der enge Zusammenhang zwischen körperlicher und psychischer Gesundheit. Die Ergebnisse der Autoren zu den untersuchten 280 Programmen zur mentalen Gesundheit zeigen, dass viele von diesen sich (lediglich) auf Erkrankungen beziehen, ohne dabei Ansätze tung aufwiesen. Die thematische Zuord- 
Tab. 1 Beiträge zu Demenzerkrankungen in der Zeitschrift für Gerontologie und Geriatrie seit $1995(n=270)$

\section{Themengebiet}

Allgemeines (u. a. Epidemiologie, Gerontotechnologie, Migration, Palliativversorgung, Prävention)

Angehörige

Demenz und geriatrische Medizin

Medikamentöse Therapie

Nichtmedikamentöse Therapien

Pflege

Neurobiologie

Psychopathologie/Gerontopsychiatrie, Testdiagnostik

Recht und Ethik

Versorgungsforschung

Beiträge ( $n)$

68

23

35

13

25

22

10

34

17

23

Tab. 2 Themenschwerpunkte zu Demenzerkrankungen in den letzten 5 Jahrgängen der Zeit schrift für Gerontologie und Geriatrie

\section{Themenschwerpunkt}

Demenz im Akutkrankenhaus

Komorbide Erkrankungen bei Demenz

Versorgung von Menschen mit Demenz im gesellschaftlichen Wandel

Menschen und Demenz - Angebote für Betroffene und Angehörige

110 years after Auguste Deter current evidence and future perspectives in dementia care research

zu Stärkung und Unterstützung mentaler Ressourcen bei den Betroffenen $\mathrm{zu}$ adressieren. Hieraus leiten die Autoren Forschungsrichtungen $a b$, die in Deutschland (Allianz für Demenz) und auch in Europa (European Innovation Partnership on Active and Healthy Ageing platform) bereits Resonanzräume gefunden haben.

H. Frohnhofen, J. Schlitzer und N. Netzer rufen zunächst die wichtigsten Fakten zur Physiologie des Schlafs im Alter in Erinnerung. Vor diesem Hintergrund fassen sie den aktuellen Stand des Wissens zur Pathophysiologie von Schlafstörungen bei an Demenz Erkrankten zusammen und geben einen Überblick über die zur Verfügung stehenden therapeutischen Optionen. Die Komplexität der von den Autoren in fundierter Weise dargestellten pathophysiologischen Zusammenhänge macht deutlich, dass einfache, z. B. nur auf pharmakologischen Interventionen beruhende Maßnahmen meist zu kurz greifen. Weiterhin weisen Frohn- hofen et al. nachdrücklich auf die Bedeutung primärer Störungen des Schlafs hin (z. B. obstruktives Schlafapnoesyndrom, Restless-legs-Syndrom), diebei der Differenzialdiagnostik und -therapie nicht unberücksichtigt bleiben dürfen. Schließlich geben die Autoren eine prägnante Übersicht über die aktuelle Literatur zur Bedeutung von Schlafstörungen als Risikofaktoren für Demenzerkrankungen; ein Thema, das vermutlich in den kommenden Jahren für die Demenzprävention noch an Bedeutung gewinnen wird.

G. Klug und C. Jagsch berichten über ein in der Region Graz etabliertes Projekt zur gemeindenahen psychosozialen Versorgung von alten Menschen mit psychischen Störungen mit einem Fokus auf den an Demenz Erkrankten. Die Autoren beschreiben die Grundvoraussetzungen für Versorgungsmodelle dieser Art und heben neben dem breiten Spektrum der für eine individualisierte Betreuung der Klienten erforderlichen Angebote die besondere Bedeutung eines per- sonenzentrierten Ansatzes hervor. Die vorgelegten Daten beschreiben den beachtlichen zeitlichen Aufwand, der in der längerfristigen psychosozialen Versorgung von Menschen mit Demenz zu leisten ist. Dieser wird aber neben dem Gewinn an Lebensqualität - auch bei ökonomischer Betrachtung - dadurch aufgewogen, dass die Betroffenen länger als in einem konventionellen Setting in einer selbstbestimmten Wohnsituation verbleiben können.

Schließlich war es uns wichtig, den Fokus auch auf die Perspektive der Betroffenen - Menschen mit Demenz und ihre Angehörigen - zu lenken. R. Schaub und $H$. von Lützau-Hohlbein gehen auf verschiedene, in dieser Hinsicht bedeutsame aktuelle Entwicklungen ein: Diese reichen von der Frage, wie hilfreich der Begriff „Demenz" unter heutigen Vorzeichen noch ist - nicht zuletzt wegen der damit verbundenen Stigmatisierung - bis hin zu gesellschaftlichen Herausforderungen, von denen manche in den letzten Jahren in Hoffnung machender Art und Weise angegangen wurden. Den Autoren gelingt es dabei, ohne die schlimmen Auswirkungen der Demenzen für die Betroffenen zu bagatellisieren, die Bedeutung von Ansätzen, die eine Verbesserung der Lebensqualität, Stärkung erhaltener Ressourcen und bestmögliche gesellschaftliche Teilhabe verfolgen, herauszuarbeiten.

Vor dem Hintergrund neuerer Erkenntnisse, wonach etwa ein Drittel der für Demenz relevanten Risikofaktoren potenziell modifizierbar ist, nähren epidemiologische Daten die Erwartung, dass sich die Kurve der altersbezogenen Erkrankungsrate abflachen könnte [4]. Weiterhin besteht die begründete Hoffnung, dass weltweite neurobiologische und klinische Forschung zukünftig Früchte trägt und krankheitsmodifizierende Therapien entwickelt werden. Gleichwohl steht außer Zweifel, dass die Demenzen auch in den kommenden Dekaden zu den großen Herausforderungen für unsere Gesellschaft und das Gesundheitssystem zählen werden. Um es in den Worten eines vor einigen Jahren erschienenen Editorials auszudrücken: „Demenz - weshalb es sich lohnt, dem 
Thema noch einige Jahrzehnte Aufmerksamkeit zu schenken“ [3].

Für die Zeitschrift für Gerontologie und Geriatrie bedeutet dies, dass sie sich weiterhin in vielfältiger Art und Weise dem Thema „Demenz“ widmen wird. Da unser Journal, wie u. a. aus • Tab. 1 hervorgeht, inhaltlich sehr breit aufgestellt ist, bietet es ein Forum, in dem „Demenz“ in einer die Fachgrenzen übergreifenden Art und Weise behandelt werden kann. Aufgrund der wissenschaftlichen und praktischen Herausforderungen der Gegenwart und näheren Zukunft ist dabei damit $\mathrm{zu}$ rechnen, dass bestimmte Themenbereiche in den kommenden Jahren ein besonders hohes Interesse erfahren werden. Vorstellbar ist dies z. B. für Fragen der Palliativversorgung, neue Entwicklungen im Bereich der Gerontotechnologie, soziokulturelle Aspekte in einem durch weltweite $\mathrm{Mi}$ grationsbewegungen geprägten Zeitalter, Wechselwirkungen zwischen altersassoziierter Multimorbidität und Demenz, präventive Strategien u. a.m.

Über neue wissenschaftliche Erkenntnisse und Ansätze hinaus wird es dabei auch wesentlich darum zu gehen haben, wie dem komplexen Versorgungsbedarf von in besonderem Maße hilfebedürftigen alten Menschen [5] in qualifizierter, humaner und ökonomisch machbarer Form Rechnung getragen werden kann. Exemplarisch sei an präventive und therapeutische Maßnahmen in Bezug auf die Demenzen häufig begleitenden psychischen und Verhaltensstörungen erinnert, die eine personalintensive und fachlich qualifizierte Betreuung gleichermaBen erfordern [4]. Auch wenn sich bisher längst nicht alle Hoffnungen, die in neue Therapien gesetzt wurden, erfüllt haben, so ist doch hervorzuheben, dass die wachsenden Erkenntnisse zu präventiven Strategien und die Möglichkeiten, vorhandenes Wissen zu fachübergreifenden und multiprofessionellen Interventionen umzusetzen, einer immer noch verbreiteten resignativen Einstellung $\mathrm{zu}$ Demezerkrankungen entgegen stehen [4, 6].

Über unmittelbar fachbezogene Aspekte hinaus erscheint es uns schließlich auch wichtig, zentrale Grundhaltungen nicht aus dem Blick zu verlieren, die wir mit unserer interdisziplinären fachlichen Expertise in den allgemeinen gesellschaftlichen Diskurs einbringen müssen. Diese betreffen z. B. ein weithin sehr negativ geprägtes Bild vom demenzkranken Menschen, das sich nicht selten auch in entsprechenden Redewendungen niederschlägt. Dem gilt es, eine Perspektive gegenüberzustellen, die die erhaltenen Ressourcen und die personale Würde der Erkrankten ins Blickfeld rückt und damit ihrer Stigmatisierung entgegenwirkt. In diesem Kontext sollten wir auch diskutieren, welche Spielräume für Selbstbestimmung für Menschen mit Demenz, z. B. in medizinischen Fragen [1], aber auch darüber hinaus im Sinne gesellschaftlicher Partizipation ermöglicht werden können [2].

Unser Dank gilt allen, die an diesem Schwerpunkt mitgewirkt haben, natürlich den Autorinnen und Autoren, der Redaktion und dem Lektorat, ganz wesentlich aber auch den diese Zeitschrift tragenden Fachgesellschaften (DGGG, DGG, ÖGGG), dem Bundesverband Geriatrie sowie den Verlagen (Springer Medizin Verlag und davor Steinkopff Verlag), die die unabdingbaren wirtschaftlichen und infrastrukturellen Rahmenbedingungen für diese wissenschaftliche Zeitschrift gewährleisten. Wir wünschen allen Beteiligten in den kommenden Jahren und Jahrzehnten Erfolg (und Freude) bei ihrer Arbeit im Sinne der Zielsetzung der Zeitschrift für Gerontologie und Geriatrie.

Ihre
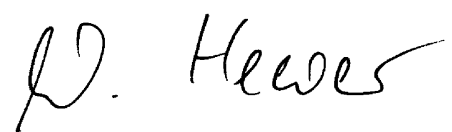

Walter Hewer und

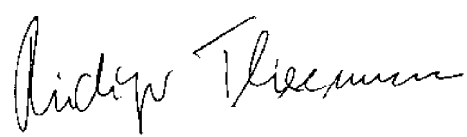

Rüdiger Thiesemann

\section{Korrespondenzadresse}

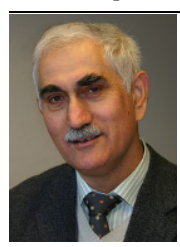

Prof. Dr. med. W. Hewer

Klinikum Christophsbad

Faurndauer Str. 6-28, 73035,

Göppingen, Deutschland

walter.hewer@

christophsbad.de

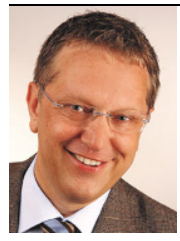

Dr. med. R. Thiesemann, MSc

Wissenschaftliches Büro Hamburg-Amerika-

Straße 15, 27472 Cuxhaven,

Deutschland

rthiesemann@web.de

Interessenkonflikt. W. Hewer und R. Thiesemann geben an, dass kein Interessenkonflikt besteht.

\section{Literatur}

1. American Geriatrics Society Expert Panel on the Care of Older Adults with Multimorbidity (2012) Guiding principles for the care of older adults with multimorbidity: an approach for clinicians. J Am Geriatr Soc 60:E1-E25. https://doi.org/10.1111/j. 1532-5415.2012.04188.x

2. Deutscher Ethikrat (2012) Demenz und Selbstbestimmung - Stellungnahme. Berlin. http://www. ethikrat.org/.Zugegriffen:30.Aug. 2017

3. Förstl H (2012) Demenz - Weshalb es sich lohnt, dem Thema noch einige Jahrzehnte Aufmerksamkeit zu schenken. Z Gerontol Geriat 45:5-5

4. Livingston $G$, Sommerlad A, Orgeta $V$ et al (2017) Dementia prevention, intervention and care. Lancet. https://doi.org/10.1016/S01406736(17)31363-6. Published Online July 20, 2017

5. Stein J, Pabst A, Luck T, Lühmann D, Riedel-Heller SG (2017) AgeCoDe and AgeQualiDe Study Group. Unmet care needs in the oldest old primary care patients with cognitive disorders: results of the AgeCoDe and AgeQualiDe Study. Dement Geriatr Cogn Disord 44(1-2):71-83

6. Winblad B, Amouyel P, Andrieu S et al (2016) Defeating Alzheimer's disease and other dementias: a priority for European science and society. Lancet Neurol 15:455-532 